This is an electronic reprint of the original article. This reprint may differ from the original in pagination and typographic detail.

Author(s): Langer, Ulrich; Repin, Sergey; Samrowski, Tatiana

Title: $\quad$ A posteriori estimates for a coupled piezoelectric model

Year: $\quad 2017$

Version:

Please cite the original version:

Langer, U., Repin, S., \& Samrowski, T. (2017). A posteriori estimates for a coupled piezoelectric model. Russian Journal of Numerical Analysis and Mathematical Modelling, 32(4), 259-266. https://doi.org/10.1515/rnam-2017-0025

All material supplied via JYX is protected by copyright and other intellectual property rights, and duplication or sale of all or part of any of the repository collections is not permitted, except that material may be duplicated by you for your research use or educational purposes in electronic or print form. You must obtain permission for any other use. Electronic or print copies may not be offered, whether for sale or otherwise to anyone who is not an authorised user. 


\title{
A Posteriori Estimates for a Coupled Piezoelectric Model
}

\author{
U. Langer * and S. Repin ${ }^{\dagger}$ and T. Samrowski ${ }^{\dagger}$
}

22nd April 2017

\begin{abstract}
The paper is concerned with a coupled problem describing piesoelectric effects in an elastic body. For this problem, we deduce majorants of the distance between the exact solution and any approximation in the respective energy class of functions satisfying the boundary conditions. The majorants are fully computable and does not contain mesh dependent constants. They vanish if and only if an approximate solution coincides with the exact one and provide a realistic measure of the accuracy in terms of the natural energy norm associated with the coupled problem studied.
\end{abstract}

Keywords: coupled systems of partial differential equations, piezoelectricity problem, a posteriori error estimates.

Mathematics Subject Classification: 35J20, 65N15, 65N30

\section{Introduction}

Mathematical models arising in the majority of applications are intended to describe the so-called multiphysics problems, which involve several equations of different types related to different processes or phenomena. Pieizoelectric models form an important class of such problems with bidirectional coupling between the mechanical and electrical system. These models are highly important for modern technological systems that transform (in a macro or micro scale) mechanical loadings into electric effects and vice versa. The first linear mathematical model and the corresponding system of differential equations of an elastic medium taking into account the interaction of electric and mechanical fields was derived by W. Voigt [19]. Later R. Toupin, R. Mindlin, L. Knopoff, S. Kaliski and J. Petikiewicz presented more advanced models of an elastic medium with polarization (see [7], [8], [17], and [18]). The effects evoked by thermal and magnetic fields are considered in [2], [3] and [6]. In [1], the authors

\footnotetext{
* Johann Radon Institute for Computational and Applied Mathematics (RICAM), Austrian Academy of Sciences Altenberger Str. 69, 4040 Linz, Austria; E-mail: ulrich.langer@assoc.oeaw.ac.at

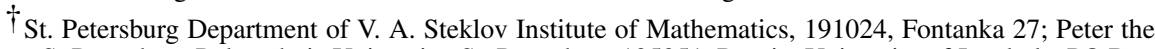
Great St.Petersburg Polytechnic University, St. Petersburg 195251, Russia; University of Jyvskulä, PO Box 35, FI-40014, Finland; E-mail: repin@pdmi.ras.ru

¥School of Engineering, Zurich University of Applied Sciences, Technikumstrasse 9, CH-8401 Winterthur, Switzerland; E-mail: samo@zhaw.ch
} 
considered a linear model (without the hysteresis effects) for the interaction of the elastic and electrical fields in a three-dimensional piezoelectric matrix with metallic inclusions.

Typically, numerical solution of multiphysics problems consists of consequent solving the respective equations by a certain splitting scheme that exchanges the data in a suitable order. This is the basic procedure used in lab and industrial environments wherein specialized single-physics solvers have been developed over the years. Difficulties of this approach lie within choosing parameters for the involved solvers that guarantee adequate overall accuracy and efficient use of computational resources. In other words, the challenging problem in quantitative analysis of complicated coupled systems is the reliability of numerical results. To address this contention it is required to understand how to compute the error in a desired quantity of interest. This is the main goal of the paper where we derive new a posteriori estimates (error majorants) for the coupled problem considered. Our method is based on a posteriori error estimates of functional type that was introduced in [9] and developed in [10], [11], [13], [15], [14] and many other publications. Error estimates of this type are derived by purely functional methods and provide fully computable measures of the difference between the exact solution a boundary value problem and an arbitrary function (approximation) from the corresponding energy space. They do not attract specific information on the approximate solution (e.g., Galerkin orthogonality, structure of meshes, numerical method used) and do not require extra regularity of the exact solution. As a result, the estimates contain no mesh dependent constants and are valid for any conforming approximation from the respective class of admissible functions. Moreover, error majorants are nonnegative functionals, which vanish if and only if an approximate solution coincides with the exact one. Therefore, they provide computable measures of the accuracy for a wide spectrum of problems (practical applications of them are systematically discussed in [5]). In this paper, we deduce error majorants of this type for the coupled piezoelectric system of elliptic type.

In Section 2, we introduce the notation and define the problem. The main result is presented in Theorem 3.1 of Section 3, which states the respective error majorant. Section 4 deals with a special but important case of isotropic media. Finally, Section 5 contains a numerical example which illustrates the performance of the error majorant.

\section{Statement of the problem and notation}

Let $\Omega$ be a bounded Lipschitz domain in $\mathbb{R}^{d}(d \in\{2,3\})$. We denote two primary fields as follows:

- $\mathbf{u}: \Omega \rightarrow \mathbb{R}^{d}$ is the vector of elastic displacement,

- $\varphi: \Omega \rightarrow \mathbb{R}$ is the scalar electric potential field.

The strain tensor $\varepsilon$ is the symmetric part of the displacement gradient:

$$
\varepsilon(\mathbf{u})=\frac{1}{2}\left(\nabla \mathbf{u}+(\nabla \mathbf{u})^{T}\right)
$$


We consider the system of equations describing deformation of a piesocristallic body in $\Omega$

$$
\begin{aligned}
\operatorname{Div} \sigma(\mathbf{u}, \varphi) & =f, \\
-\operatorname{div} D(\mathbf{u}, \varphi) & =g,
\end{aligned}
$$

containing the body force vector $f$ and the scalar field of the electric charge density $g$. Here Div and div denote the divergence operators for the tensor and vector valued functions, respectively, i.e., $\operatorname{Div} \tau=\nabla \cdot \tau=\tau_{i j, j}$ and $\operatorname{div} \mathbf{q}=\nabla \cdot \mathbf{q}=q_{i, i}$. Here and later on the Einstein summation convention of summation over the repeated indices is adopted.

The stress tensor $\sigma$ and the dielectric displacement $D$ are coupled via the linear piezoelectric material law:

$$
\begin{aligned}
\sigma(\mathbf{u}, \varphi) & =\mathbb{L} \varepsilon(\mathbf{u})+B \cdot \nabla \varphi \\
-D(\mathbf{u}, \varphi) & =K \cdot \nabla \varphi-B^{T}: \varepsilon(\mathbf{u})
\end{aligned}
$$

In (2.4) and (2.5), $\mathbb{L}=\left(L_{i j k l}\right)$ is the (forth-order) linear-elastic material tensor, which is subject to the condition

$$
c_{1}^{2}|\varepsilon|^{2} \leqslant \mathbb{L} \varepsilon: \varepsilon \leqslant c_{2}^{2}|\varepsilon|^{2}, \quad \forall \varepsilon \in \mathbb{M}_{\text {sym }}^{d \times d},
$$

where $\mathbb{M}_{s y m}^{d \times d}$ is the space of symmetric real valued $d \times d$ tensors. We assume that the elements of the elasticity tensor are bounded and possess natural symmetry properties:

$$
\mathbb{L}_{i j k m}=\mathbb{L}_{j i k m}=\mathbb{L}_{k m i j} \in L^{\infty}(\Omega), \quad i, j, k, m=1, \ldots, d .
$$

Also, the relations (2.4) and (2.5) contain the (third-order) piezoelectric tensor

$$
B=\left(b_{i j s}\right), b_{i j s} \in L^{\infty}(\Omega)
$$

and the (second-order) dielectric material tensor $K=\left(K_{i j}\right)$,, which satisfies the symmetry and ellipticity conditions

$$
\begin{aligned}
& K_{i j}=K_{j i} \in L^{\infty}(\Omega), \\
& \gamma_{1}^{2}|\zeta|^{2} \leqslant K \zeta \cdot \zeta \leqslant \gamma_{2}^{2}|\zeta|^{2}, \quad \forall \zeta \in \mathbb{R}^{d} .
\end{aligned}
$$

The system (2.2) - (2.5) is supplied with the boundary conditions

$$
\begin{array}{lll}
\mathbf{u}=\mathbf{u}_{0} & \text { on } & \Gamma_{D, \mathbf{u}}, \\
\varphi=\varphi_{0} & \text { on } & \Gamma_{D, \varphi}
\end{array}
$$

stated for the case where the Dirichlet boundary conditions $\mathbf{u}_{0}$ for the elastic component of the solution is defined on $\Gamma_{D, \mathbf{u}}$ and $\varphi=\varphi_{0}$ for the electric component is 
defined on $\Gamma_{D, \varphi}$ (in general, these are two different parts of the boundary $\Gamma$ ). On the remaining parts $\Gamma_{N, \mathbf{u}}$ and $\Gamma_{N, \varphi}$ we impose the homogeneous Neumann conditions

$$
\begin{array}{lll}
\sigma \cdot \mathbf{n}=0 & \text { on } & \Gamma_{N, \mathbf{u}}, \\
D \cdot \mathbf{n}=0 & \text { on } & \Gamma_{N, \varphi} .
\end{array}
$$

In what follows, we use the spaces $H(\Omega$, Div $)$ and $H(\Omega$, div $)$ containing square summable tensor valued and vector valued functions having square summable divergences. Subspaces of these spaces formed by the functions satisfying the homogeneous Neumann conditions are denoted by

$$
H^{+}(\Omega, \text { Div }):=\left\{\tau \in H(\Omega, \text { Div }) \mid \int_{\Omega}(\tau: \nabla \mathbf{w}+\operatorname{Div} \tau \cdot \mathbf{w}) d x=0 \text { on } \Gamma_{N, \mathbf{u}} \forall \mathbf{w} \in V_{0},\right\}
$$

and

$H^{+}(\Omega, \operatorname{div}):=\left\{\mathbf{q} \in H(\Omega, \operatorname{div}) \mid \int_{\Omega}(q \cdot \nabla \psi+\operatorname{div} q \psi) d x=0\right.$ on $\left.\Gamma_{N, \varphi} \quad \forall \psi \in M_{0}\right\}$,

respectively.

The generalized solution of the problem (2.2)-(2.13) is defined by the system of integral identities

$$
\begin{aligned}
c(\mathbf{u}, \mathbf{w})+b(\varphi, \mathbf{w})=F(\mathbf{w}), & \forall w \in V_{0}, \\
-b(\eta, \mathbf{u})+k(\varphi, \eta)=G(\eta), & \forall \eta \in M_{0},
\end{aligned}
$$

where the solution pair $(\mathbf{u}, \varphi)$ is seeking in the sets

$$
\mathbf{u} \in V_{0}+\mathbf{u}_{\mathbf{0}}, V_{0}:=\left\{\mathbf{v} \in H^{1}\left(\Omega ; \mathbb{R}^{3}\right)|\mathbf{v}|_{\Gamma_{D, \mathbf{u}}}=0\right\}
$$

and

$$
\varphi \in M_{0}+\varphi_{0}, M_{0}:=\left\{\psi \in H^{1}(\Omega)|\psi|_{\Gamma_{D, \varphi}}=0\right\} .
$$

The right hand sides and forms associated with elasticity, piezoelectric and permittivity tensors are defined by the relations

$$
\begin{aligned}
c(\mathbf{u}, \mathbf{w}) \quad:=\int_{\Omega} \varepsilon(\mathbf{u}): \mathbb{L}: \varepsilon(\mathbf{w}) \mathrm{d} \mathbf{x}=\int_{\Omega} L_{i j k l} \varepsilon_{i j}(\mathbf{u}): \varepsilon_{k l}(\mathbf{w}) \mathrm{d} \mathbf{x}, \\
b(\varphi, \mathbf{w}) \quad:=\int_{\Omega} \varepsilon(\mathbf{w}): B \cdot \nabla \varphi \mathrm{d} \mathbf{x}=\int_{\Omega} b_{i j s} \varepsilon_{i j}(\mathbf{u}) \varphi_{, s} \mathrm{~d} \mathbf{x}, \\
k(\varphi, \eta) \quad:=\int_{\Omega} \nabla \varphi \cdot K \cdot \nabla \eta \mathrm{d} \mathbf{x}=\int_{\Omega} K_{i j} \varphi_{, i} \eta_{, j} \mathrm{~d} \mathbf{x}, \\
F(\mathbf{w}):=\int_{\Omega} f \cdot \mathbf{w} \mathrm{d} \mathbf{x}, \quad G(\eta):=\int_{\Omega} g \eta \mathrm{d} \mathbf{x},
\end{aligned}
$$


Solution of the system (2.14)-(2.15) exists and it is a unique element of the set $\left(V_{0}+\right.$ $\left.\mathbf{u}_{0}\right) \times\left(M_{0}+\varphi_{0}\right)[16]:$

It is not difficult to see that the norm

$$
\mid[\mathbf{u}, \varphi \mid]^{2}:=\|\varepsilon(\mathbf{u})\|_{\mathbb{L}}^{2}+\|\nabla \varphi\|_{K}^{2}=c(\mathbf{u}, \mathbf{u})+k(\varphi, \varphi)
$$

is the natural energy norm associated with our problem. We use it as a suitable measure of the distance to the exact solution.

\section{Estimate of the deviation from the exact solution}

Assume that $\mathbf{v} \in V_{0}+\mathbf{u}_{0}$ and $\psi \in M_{0}+\varphi_{0}$ are approximations of $\mathbf{u}$ and $\varphi$, respectively. Our goal is to deduce a computable upper bound of the norm

$$
\mid[\mathbf{u}-\mathbf{v}, \varphi-\psi \mid]^{2}:=\|\varepsilon(\mathbf{u}-\mathbf{v})\|_{\mathbb{L}}^{2}+\|\nabla(\varphi-\psi)\|_{K}^{2} .
$$

Consider the quantities

$$
\mathscr{M}_{1}(\mathbf{v}, \psi, \tau):=\|\tau-\mathbb{L} \varepsilon(\mathbf{v})-B \cdot \nabla \psi\|_{\mathbb{L}^{-1}}+\mu_{F}\left(\mathbb{L}, \Omega, \Gamma_{D, \mathbf{u}}\right)\|f+\operatorname{Div} \tau\|
$$

and

$$
\mathscr{M}_{2}(\mathbf{v}, \psi, q):=\left\|\mathbf{q}-K \nabla \psi+B^{T}: \varepsilon(\mathbf{v})\right\|_{K^{-1}}+\mu_{F}\left(K, \Omega, \Gamma_{D, \varphi}\right)\|g+\operatorname{div} \mathbf{q}\|,
$$

where $\mu_{F}$ are constants in the Friedrichs type inequalities

$$
\|\mathbf{w}\| \leqslant \mu_{F}\left(\mathbb{L}, \Omega, \Gamma_{D, \mathbf{u}}\right)\|\varepsilon(\mathbf{w})\|_{\mathbb{L}}, \quad \forall \mathbf{w} \in V_{0}
$$

and

$$
\|\eta\| \leqslant \mu_{F}\left(K, \Omega, \Gamma_{D, \varphi}\right)\|\nabla \eta\|_{K}, \quad \forall \varphi \in M_{0} .
$$

Here and later on $\|\cdot\|$ stands for the $L^{2}$ norm of a vector or scalar valued function. The quantities $\mathscr{M}_{1}$ and $\mathscr{M}_{2}$ contain only known functions (approximations $\mathbf{v}$ and $\psi$, and the functions $\tau \in H^{+}\left(\Omega\right.$, Div) and $q \in H^{+}(\Omega$, div) that can be viewed as approximations of the exact elastic stress and of the exact flux, respectively).

Theorem below shows that these two quantities majorate the error norm.

Theorem 3.1. $\quad$ i) For any $\mathbf{v} \in V_{0}+\mathbf{u}_{0}$ and $\psi \in M_{0}$ combined error norm is bounded from above by the estimate

$$
|[\mathbf{u}-\mathbf{v}, \varphi-\psi]|^{2} \leqslant \mathscr{M}_{1}^{2}(\mathbf{v}, \psi, \tau)+\mathscr{M}_{2}^{2}(\mathbf{v}, \psi, \mathbf{q}),
$$

where $\tau$ and $q$ are arbitrary functions in the spaces $H^{+}\left(\Omega\right.$, Div) and $H^{+}(\Omega$, div $)$, respectively.

ii) The right-hand side of (3.4) vanishes if and only if $\mathbf{v}=\mathbf{u}, \psi=\varphi, \tau=\mathbb{L} \varepsilon(\mathbf{u})+$ $B \cdot \nabla \varphi$ and $\mathbf{q}=K \nabla \varphi-B^{T}: \varepsilon(\mathbf{u})$. 
Proof: Let $v \in V_{0}+u_{0}$ and $\psi \in M_{0}+\varphi_{0}$. We reform (2.14) and (2.15) as follows

$$
\begin{aligned}
c(\mathbf{u}-\mathbf{v}, \mathbf{w})+b(\varphi-\psi, \mathbf{w}) & =F(\mathbf{w})-c(\mathbf{v}, \mathbf{w})-b(\psi, \mathbf{w}) \\
-b(\eta, \mathbf{u}-\mathbf{v})+k(\varphi-\psi, \eta) & =G(\eta)+b(\eta, \mathbf{v})-k(\psi, \eta) .
\end{aligned}
$$

Let $\mathbf{w}=\mathbf{u}-\mathbf{v}$ and $\eta=\varphi-\psi$. By adding the equations (3.5) and (3.6), we obtain the norm $\mid[\mathbf{u}-\mathbf{v}, \varphi-\psi \mid]^{2}$ in the left-hand side.

In the right-hand side, we have

$$
\begin{aligned}
& F(\mathbf{w})-c(\mathbf{v}, \mathbf{w})-b(\psi, \mathbf{w})+G(\eta)+b(\eta, \mathbf{v})-k(\psi, \eta) \\
&=\int_{\Omega}(f+\operatorname{Div} \tau) \cdot \mathbf{w} \mathrm{d} \mathbf{x}+\int_{\Omega}(\tau-\mathbb{L} \varepsilon(\mathbf{v})-B \cdot \nabla \psi): \varepsilon(\mathbf{w}) \mathrm{d} \mathbf{x} \\
& \quad+\int_{\Omega}(g+\operatorname{div} \mathbf{q}) \eta \mathrm{d} \mathbf{x}+\int_{\Omega}\left(\mathbf{q}-K \nabla \psi+B^{T}: \varepsilon(\mathbf{v})\right) \cdot \nabla \eta \mathrm{d} \mathbf{x}
\end{aligned}
$$

where $\tau \in H^{+}\left(\Omega\right.$, Div) and $\mathbf{q} \in H^{+}(\Omega$, div $)$. The first term is estimated as follows:

$$
\int_{\Omega}(f+\operatorname{Div} \tau) \cdot \mathbf{w} \mathrm{d} \mathbf{x} \leqslant\|f+\operatorname{Div} \tau\|\|\mathbf{w}\| \leqslant \mu_{F}\left(\mathbb{L}, \Omega, \Gamma_{D, \mathbf{u}}\right)\|\varepsilon(\mathbf{w})\|_{\mathbb{L}}\|f+\operatorname{Div} \tau\| .
$$

Analogously

$$
\int_{\Omega}(g+\operatorname{div} q) \eta \mathrm{d} \mathbf{x} \leqslant\|g+\operatorname{div} \mathbf{q}\| \mu_{F}\left(K, \Omega, \Gamma_{D, \varphi}\right)\|\nabla \eta\|_{K} .
$$

Next, we use the algebraic inequalities

$$
\chi: \varepsilon \leqslant(\mathbb{L} \chi: \chi)^{1 / 2}\left(\mathbb{L}^{-1} \varepsilon: \varepsilon\right)^{1 / 2}
$$

and

$$
\mathbf{q} \cdot p \leqslant(K \mathbf{q} \cdot \mathbf{q})^{1 / 2}\left(K^{-1} p \cdot p\right)^{1 / 2}
$$

Hence,

$$
\begin{aligned}
& \int_{\Omega}(\tau-\mathbb{L} \varepsilon(\mathbf{v})-B \cdot \nabla \psi): \varepsilon(\mathbf{w}) \mathrm{d} \mathbf{x} \leqslant\|\tau-\mathbb{L} \varepsilon(\mathbf{v})-B \cdot \nabla \psi\|_{\mathbb{L}^{-1}}\|\varepsilon(\mathbf{w})\|_{\mathbb{L}} \\
& \int_{\Omega}\left(\mathbf{q}-K \nabla \psi+B^{T}: \varepsilon(\mathbf{v})\right) \cdot \nabla \eta \mathrm{d} \mathbf{x} \leqslant\left\|\mathbf{q}-K \nabla \psi+B^{T}: \varepsilon(\mathbf{v})\right\|_{K^{-1}}\|\nabla \eta\|_{K} .
\end{aligned}
$$

In the right-hand side we have two terms:

$$
\begin{aligned}
& I_{1}=\left(\mu_{F}\left(\mathbb{L}, \Omega, \Gamma_{D, \mathbf{u}}\right)\|f+\operatorname{Div} \tau\|+\|\tau-\mathbb{L} \varepsilon(\mathbf{v})-B \cdot \nabla \psi\|_{\mathbb{L}^{-1}}\right)\|\varepsilon(\mathbf{u}-\mathbf{v})\|_{\mathbb{L}}, \\
& I_{2}=\left(\mu_{F}\left(K, \Omega, \Gamma_{D, \varphi}\right)\|g+\operatorname{div} \mathbf{q}\|+\left\|\mathbf{q}-K \nabla \psi+B^{T}: \varepsilon(\mathbf{v})\right\|_{K^{-1}}\right)\|\nabla(\varphi-\psi)\|_{K}
\end{aligned}
$$


Then,

$$
|[\mathbf{u}-\mathbf{v}, \varphi-\psi]|^{2} \leqslant I_{1}+I_{2} \leqslant\left(\mathscr{M}_{1}^{2}(\mathbf{v}, \psi, \tau)+\mathscr{M}_{2}^{2}(\mathbf{v}, \psi, q)\right)^{1 / 2}|[\mathbf{u}-\mathbf{v}, \varphi-\psi]|,
$$

and we arrive at the estimate (3.4).

To prove $i$ ), we note that vanishing of the right-hand side of (3.4) means that almost everywhere in $\Omega$ it holds

$$
\begin{aligned}
& \operatorname{Div} \tau+f=0, \\
& \operatorname{div} \mathbf{q}+g=0, \\
& \tau=\mathbb{L} \varepsilon(\mathbf{v})+B \cdot \nabla \psi, \\
& \mathbf{q}=K \nabla \psi-B^{T}: \varepsilon(\mathbf{v}) .
\end{aligned}
$$

From (3.9) and (3.11) it follows that:

$$
\int_{\Omega}(\mathbb{L} \varepsilon(\mathbf{v})+B \cdot \nabla \psi): \varepsilon(\mathbf{w}) \mathrm{d} \mathbf{x}=\int_{\Omega} f \cdot \mathbf{w} \mathrm{d} \mathbf{x} \quad \forall \mathbf{w} \in V_{0} .
$$

Analogously, (3.10) and (3.12) imply

$$
\int_{\Omega}(K \nabla \psi \cdot \nabla \eta-\varepsilon(\mathbf{v}): B \cdot \nabla \eta) \mathrm{d} \mathbf{x}=\int_{\Omega} g \eta \mathrm{d} \mathbf{x} \quad \forall \eta \in M_{0} .
$$

Since $\mathbf{v}$ and $\psi$ satisfy the main boundary conditions, (3.13) and (3.14) show that they satisfy the system (2.14) - (2.15), which solution is unique. Thus, we conclude these functions coincide with $\mathbf{u}$ and $\varphi$, respectively.

Remark 3.1. It is easy to see that

$$
\begin{aligned}
& \mu_{F}\left(\mathbb{L}, \Omega, \Gamma_{D, \mathbf{u}}\right) \leqslant\|\mathbb{L}\| \mu_{F}\left(\Omega, \Gamma_{D, \mathbf{u}}\right), \\
& \mu_{F}\left(K, \Omega, \Gamma_{D, \varphi}\right) \leqslant\|K\| \mu_{F}\left(\Omega, \Gamma_{D, \varphi}\right),
\end{aligned}
$$

where $\mu_{F}\left(\Omega, \Gamma_{D, \mathbf{u}}\right)$ and $\mu_{F}\left(\Omega, \Gamma_{D, \varphi}\right)$ are constants in the inequalities

$$
\begin{array}{ll}
\|\mathbf{w}\| \leqslant \mu_{F}\left(\Omega, \Gamma_{D, \mathbf{u}}\right)\|\nabla \mathbf{w}\|, & \forall \mathbf{w} \in V_{0}, \\
\|\eta\| \leqslant \mu_{F}\left(\Omega, \Gamma_{D, \varphi}\right)\|\nabla \eta\|, & \forall \varphi \in M_{0} .
\end{array}
$$

Computable estimates of the constants $\mu_{F}\left(\Omega, \Gamma_{D, \mathbf{u}}\right)$ and $\mu_{F}\left(\Omega, \Gamma_{D, \varphi}\right)$ can be found in [4], [11], [12].

It is worth adding a concise comment on application of the majorant in combination with finite element approximations. Let $\mathbf{v}=\mathbf{u}_{h}$ and $\psi=\varphi_{h}$, be FEM solutions computed on a mesh $\mathscr{T}_{h}$. The simplest way to apply (3.4) is to reconstruct $\tau$ and $\mathbf{q}$ from the respective numerical stress $\widehat{\tau}_{h}=\mathbb{L} \varepsilon\left(\mathbf{u}_{h}\right)$ and numerical flux $\widehat{\mathbf{q}}_{h}=K \nabla \psi_{h}$. In 
general, these functions may not belong to $H^{+}\left(\Omega\right.$, Div) and $H^{+}(\Omega$, div), respectively. Therefore, a certain averaging (smoothing) procedure is required. A wide spectrum of known type post-processing procedures can be used for this purpose (see, e.g., [?,?] and the references therein). Post-processed functions $\widehat{\tau}_{h}$ and $\widehat{\mathbf{q}}_{h}$ preserve continuity of the normal components $\tau_{h} \mathbf{n}$ and $(\mathbf{q} \cdot \mathbf{n})$ along the interelement boundaries. The substitution of $\tau_{h}$ and $\mathbf{q}_{h}$ will give a simply computable and guaranteed bound of the error. To make a sharper bound it is necessary to minimize the right-hand side of (3.4) over $\tau$ and $\mathbf{q}$ using $\widehat{\tau}_{h}$ and $\widehat{\mathbf{q}}_{h}$ as the initial guess. Since $\mathscr{M}_{1}^{2}+\mathscr{M}_{2}^{2}$ is a quadratic functional, this can be done by standard procedures well tested for elliptic boundary value problems (see [5]).

In the last section, we discuss numerical evaluation of the majorant with the paradigm of a model plane strain problem associated with isotropic media.

\section{Isotropic media in 3D}

If elastic media is isotropic, then the components of $\mathbb{L}$ and $\mathbb{L}^{-1}$ depend on two material constants only $K_{0}$ and $\mu$ that depend on properties of media to resist compression and shear forces, respectively. Due to $\varepsilon=\frac{1}{3} \operatorname{tr} \varepsilon \mathbb{I}+\varepsilon^{D}$ and $\operatorname{tr} \varepsilon=\varepsilon_{11}+\varepsilon_{22}+\varepsilon_{33}$, we obtain in this case,

$$
\mathbb{L} \varepsilon=K_{0} \operatorname{tr} \varepsilon \mathbb{I}+2 \mu \varepsilon^{D}
$$

and

$$
\mathbb{L}^{-1} \tau=\frac{1}{9 K_{0}} \operatorname{tr} \tau \mathbb{I}+\frac{1}{2 \mu} \tau^{D}
$$

Then

$$
\mathbb{L} \varepsilon+B \nabla \psi-\tau=\left(K_{0} \operatorname{tr} \varepsilon+B_{0} \operatorname{tr} \Psi-\frac{1}{3} \operatorname{tr} \tau\right) \mathbb{I}+\left(2 \mu \varepsilon^{D}+v \Psi^{D}-\tau^{D}\right),
$$

where

$$
B_{0}=\frac{b_{13}}{3}=\frac{b_{23}}{3}=\frac{b_{33}}{3}, \quad v=\frac{b_{42}}{2}=\frac{b_{51}}{2}, \quad \Psi=\left(\begin{array}{ccc}
\psi_{, 3} & 0 & \psi_{, 1} \\
0 & \psi_{, 3} & \psi_{, 2} \\
\psi_{, 1} & \psi_{, 2} & \psi_{, 3}
\end{array}\right),
$$

and we find

$$
\begin{aligned}
\varepsilon(v)+\mathbb{L}^{-1}(B \nabla \psi)-\mathbb{L}^{-1} \tau=( & \left.\frac{1}{3} \operatorname{tr} \varepsilon+\frac{B_{0}}{3 K_{0}} \operatorname{tr} \Psi-\frac{1}{9 K_{0}} \operatorname{tr} \tau\right) \mathbb{I} \\
& +\left(\varepsilon^{D}+\frac{v}{2 \mu} \Psi^{D}-\frac{1}{2 \mu} \tau^{D}\right) .
\end{aligned}
$$


Then, the parts $\mathscr{M}_{1}$ and $\mathscr{M}_{2}$ of the majorant (3.4) have the form

$$
\begin{aligned}
\mathscr{M}_{1} & =\left(\int_{\Omega}\left[\frac{K_{0}}{3}\left(\operatorname{tr} \varepsilon+\frac{B_{0}}{K_{0}} \operatorname{tr} \Psi-\frac{1}{3 K_{0}} \operatorname{tr} \tau\right)^{2}+2 \mu\left|\varepsilon^{D}+\frac{v}{2 \mu} \Psi^{D}-\frac{1}{2 \mu} \tau^{D}\right|^{2}\right]\right)^{1 / 2} \\
& +\mu_{F}\left(\mathbb{L}, \Omega, \Gamma_{D, \mathbf{u}}\right)\|f+\operatorname{Div} \tau\| \\
\mathscr{M}_{2} & =\left(\int_{\Omega}\left(\begin{array}{c}
\frac{q_{2}+4 v \varepsilon_{23}}{k_{22}}-\psi_{, 2} \\
\frac{q_{3}+3 B_{0} \operatorname{tr} \varepsilon}{k_{33}}-\psi_{, 3}
\end{array}\right) \cdot\left(\begin{array}{c}
q_{1}+4 v \varepsilon_{13}-k_{11} \psi_{, 1} \\
q_{2}+4 v \varepsilon_{23}-k_{22} \psi_{, 2} \\
q_{3}+3 B_{0} \operatorname{tr} \varepsilon-k_{33} \psi_{, 3}
\end{array}\right)\right)^{1 / 2} \\
& +\mu_{F}\left(K, \Omega, \Gamma_{D, \varphi}\right)\|g+\operatorname{divq}\| .
\end{aligned}
$$

Due to symmetries in the elasticity, the piezoelectric and the permittivity tensors, we can use the so-called Voigt notation (or Voigt mapping), that suggests a method of compact representation of symmetric tensors (this leads to simplifications in writing certain algebraic relations). For example, the tensors from (2.18)-(2.20) can be expressed as matrices

$$
\mathbb{L}=\left(\begin{array}{cccccc}
c_{11} & c_{12} & c_{13} & 0 & 0 & 0 \\
c_{12} & c_{22} & c_{23} & 0 & 0 & 0 \\
c_{13} & c_{23} & c_{33} & 0 & 0 & 0 \\
0 & 0 & 0 & c_{44} & 0 & 0 \\
0 & 0 & 0 & 0 & c_{55} & 0 \\
0 & 0 & 0 & 0 & 0 & c_{66}
\end{array}\right), B=\left(\begin{array}{ccc}
0 & 0 & b_{13} \\
0 & 0 & b_{23} \\
0 & 0 & b_{33} \\
0 & b_{42} & 0 \\
b_{51} & 0 & 0 \\
0 & 0 & 0
\end{array}\right), K=\left(\begin{array}{ccc}
k_{11} & 0 & 0 \\
0 & k_{22} & 0 \\
0 & 0 & k_{33}
\end{array}\right) .
$$

In the Voigt notation the strain tensor (2.1) and its deviatoric part are represented by the vectors

$$
\varepsilon=\left(\begin{array}{c}
\varepsilon_{11} \\
\varepsilon_{22} \\
\varepsilon_{33} \\
2 \varepsilon_{23} \\
2 \varepsilon_{13} \\
2 \varepsilon_{12}
\end{array}\right) \quad \text { and } \quad \varepsilon^{D}=\left(\begin{array}{c}
\frac{2, \varepsilon_{11}-\varepsilon_{22}-\varepsilon_{33}}{3} \\
\frac{2, \varepsilon_{22}-\varepsilon_{11}-\varepsilon_{33}}{3} \\
\frac{2, \varepsilon_{33}-\varepsilon_{11}-\varepsilon_{22}}{3} \\
2 \varepsilon_{23} \\
2 \varepsilon_{13} \\
2 \varepsilon_{12}
\end{array}\right)
$$

respectively. If the material (e.g., PZT ceramics) can be treated as isotropic, we obtain additionaly

$$
c_{11}=c_{22}=c_{33}=K_{0}+\frac{4}{3} \mu, \quad c_{44}=c_{55}=\mu, \quad c_{66}=\frac{c_{11}-c_{22}}{2}
$$




$$
c_{12}=c_{21}=c_{13}=c_{31}=c_{23}=c_{32}=K_{0}-\frac{2}{3} \mu,
$$

and

$$
b_{13}=b_{23}, \quad b_{42}=b_{51}, \quad k_{11}=k_{22} .
$$

In terms of the Lame constants

$$
\mathbb{L}=\left(\begin{array}{cccccc}
\lambda+2 \mu & \lambda & \lambda & 0 & 0 & 0 \\
\lambda & \lambda+2 \mu & \lambda & 0 & 0 & 0 \\
\lambda & \lambda & \lambda+2 \mu & 0 & 0 & 0 \\
0 & 0 & 0 & \mu & 0 & 0 \\
0 & 0 & 0 & 0 & \mu & 0 \\
0 & 0 & 0 & 0 & 0 & \mu
\end{array}\right)
$$

and $\mathscr{M}_{1}$ can be expressed as follows:

$$
\begin{aligned}
\mathscr{M}_{1}= & \left\{\int _ { \Omega } \left[\left(\lambda \operatorname{tr} \varepsilon+2 \rho \psi_{, 2}\right)\left(\frac{\rho \mu-v \lambda}{\mu(\lambda+\mu)} \operatorname{tr} \Psi+\frac{\lambda}{4 \mu(\lambda+\mu)} \operatorname{tr} \tau\right) \mathbb{I}\right.\right. \\
& \left.\left.+2 \mu|\varepsilon+2 v \Psi-\tau|^{2}\right]\right\}^{1 / 2}+\mu_{F}\left(\mathbb{L}, \Omega, \Gamma_{D, \mathbf{u}}\right)\|f+\operatorname{Div} \tau\|
\end{aligned}
$$

\section{References}

1. Buchukuri, T. , Chkadua, O., Natroshvili , D., and Sändig, A.-M. (2005): Solvability and regularity results to boundary-transmission problems for metallic and piezoelectric elastic materials. Berichte aus dem Institut für Angewandte Analysis und Numerische Simulation, IANS, Preprint 2005/004

2. Kaliski, S. and Patykiewicz, G. (1960): Dynamical equation of motion coupled with the field of temperature and resolving function for elastic and inelastic anisotropic bodies in the magnetic fields. Proc. Vibr. Problem, 1, 4.

3. Knopoff, L. (1955): The interaction between elastic wave motions and a magnetic field in electric conductors. J. Geophys. Res., $60,4$.

4. Kuznetsov, Yu. and Repin, S. (2003): Guaranteed lower bounds of the smallest eigenvalues of elliptic differential operators. Russian J. Numer. Anal. Math. Modelling, , 21, 2, 135-156.

5. Mali, O., Nettaanmäki, P., and Repin, S. (2014): Accuracy verification methods. Theory and algorithms. Springer, New York

6. Mindlin, R.D. (1961): On the equations of motion of piezoelectric crystals. In: J.R.M. Radok(ed): Problems of Continuum Mechanics. SIAM, 282-290.

7. Mindlin, R.D (1968): Polarization gradient in elastic dielectrics. Int. J. Solids Structures, 6.

8. Mindlin, R.D. (1972): Elasticity, piezoelasticity and crystal lattice dynamics. J. Elasticity, 2, 4.

9. Repin, S. (1997): A posteriori error estimation for nonlinear variational problems by duality theory. Zapiski Nauch. Semin. (POMI), 243, 201-214. 
10. Repin, S.(1999): A unified approach to a posteriori error estimation based on duality error majorants. Modelling '98 (Prague), Math. Comput. Simulation, 50, no.1-4, $305-321$

11. Repin, S.(2008): A posteriori estimates for partial differential equations. Radon Series on Computational and Applied Mathematics, 4. Walter de Gruyter GmbH \& Co. KG, Berlin.

12. Repin, S. (2012): Computable Majorants of constants in the Poincare and Friedrichs inequalities. J. Math. Sci., 186, 2, 307-321.

13. Repin, S. and Samrowski, T. and Sauter, S. (2012): Combined A Posteriori Modeling-Discretization Error Estimate for Elliptic Problems with Complicated Interfaces. ESAIM: Math. Model. Num. Anal, 46, 6, 1389-1405.

14. Repin, S. and Valdman, J. (2008): Functional a posteriori error estimates for problems with nonlinear boundary conditions. Journal of Numerical Mathematics, 16, 1, 51-81.

15. Samrowski, T. (2014): Combined Error Estimates in the case of the Dimension Reduction. CMAM, 14, 113-134.

16. Steinhorst, P. (2009): Anwendung adaptiver FEM für piezoelektrische und spezielle mechanische Probleme. Dissertation, Fakultät für Mathematik, Technische Universit?at Chemnitz.

17. Toupin, R.A. (1956): The elastic dielectrics. J. Rat. Mech. Analysis, 5.

18. Toupin, R.A. (1963): A dynamical theory of elastic dielectrics. Int. J. Engng. Sci., 1, 1.

19. Voigt, W. (1910): Lehrbuch der Kristall-Physik. Leipzig: Teubner. 